\title{
Instructions for Book Reviewers
}

Length

Manuscripts should be 800-1,000 words (3-4 double-spaced pages).

\section{Content}

Reviews should evaluate books as a contribution to the literature of library and information science, characterizing the content of the book and critically appraising it. Try to position the book within the intellectual history of the field by comparing it to other books or ideas that are related to it. Library Resources \& Technical Services is not a library book selection tool and does not include purchasing recommendations.

\section{Style}

LRTS follows The Chicago Manual of Style, 14th ed. (1993). Please include the following information in the review:

Header: complete bibliographic citation of the book, in the following format:

\section{Advances in Library Auto- mation and Networking, Vol. 5 (1994). Ed. Joe A. Hewitt and Charles W. Bailey, Jr. Greenwich, Conn.: JAI Pr., 1994. 282p. \$73.25 (ISBN 1- 55938-510-3).}

Quotations: give page numbers in parentheses (36) (vii) immediately following the quote.

Quotation marks around chapter or section heads: be sure these reflect the exact language used in the book.

Author(s), title, place, and date of publication of all works cited: a complete bibliographic citation should be included at the end of the text. Use the author-date system of references, as described in The Chicago Manual of Style, chapter 16. Please verify all citations carefully.

Reviewer's name, e-mail address, and affiliation immediately following the text: if unaffiliated, give city of residence.

Do not use end notes for reasons other than the citation of other works.

Use first name or initial of each person cited for the first time.

Avoid library jargon because it may become dated (e.g., online catalog, not OPAC).

In quotations, identify acronyms and initialisms (e.g., ALA, ALCTS, ASIS, etc.) with the full name at their first use: "Library of Congress (LC) policy is." Use the acronym for subsequent references to the same entity.

For good advice and helpful reminders on writing and style, check the following compendium, which includes a link to the 1918 edition of Strunk's The Elements of Style: http://andromeda.rutgers.edu/ jlynch Nriting.

\section{Format}

Reviews submitted in machine-readable form are greatly appreciated. To facilitate editing, please observe these additional formatting instructions:

- double-space all text, including headers;

- do not indent paragraphs, but use two carriage returns between paragraphs;

- do not use carriage returns at line ends (use your word processor's word-wrap capability);

- keep the right margin ragged (do not use full justification) and do not hyphenate words at the line breaks;
- do not use your word processor's automatic endnoting capability;

- treat the endnotes as the final paragraph of your review;

- include at least 1" margins on all sides.

\section{Submitting the Review}

If possible, please send your review as an e-mail attachment, preferably a Microsoft Word Document. If e-mail is not possible, send the review on a $3.5^{\prime \prime}$ disk

\section{And ... A Few More Suggestions, Especially for Novice Reviewers}

Allow enough time to read the book, think about it, draft your review, and rewrite. Don't base your review on a careful reading of the book's introduction and conclusion or-heaven forbid-the book jacket and other reviews. It is often helpful to ask a colleague to read your review and comment. If you would like to send a draft for comment before preparing the final version, feel free to do so, well in advance of the deadline.

A review is not an abstract of the book. To characterize the book's content, a synthesis or a description of its highlights is preferable to a chapterby-chapter summary. This is especially important, and also a difficult task if you are reviewing a compilation. Careful attention to this part of the review usually will help you focus on evaluating the book.

A review is an opinion piece, not a book report. State opinions clearly-be fair and specific with both praise and criticism. Understand the author's purpose in writing the book and evaluate the success in meeting stated objectives. Are the objectives 
worthwhile? How does the book compare to others of similar content and purpose? What is unique or especially valuable about the book? Is the book controversial? To what audience will it be most interesting and useful? Are there any significant errors or omissions?

\section{And One Suggestion for Everyone . . .}

Clear your mind, especially at the rewrite stage, by taking another look at Strunk and White. Your editor does!

Thank you for reviewing a book for LRTS. If you have questions about review style or about submitting your review, please contact me: Margaret Rohdy, Head of Research, Training \& Quality Management, University of Pennsylvania Libraries, 3420 Walnut St., Philadelphia, PA 19104-6206; phone: (215) 898-0314; fax: (215) 5739610; e-mail: rohdy@pobox.upenn.edu. 